\title{
Effect of reconstruction parameters on cone beam CT trabecular bone microstructure quantification in sheep
}

\author{
Aso Muhammad Ali Muhammad ${ }^{1}$, Norliza Ibrahim ${ }^{1 *}$, Rohana Ahmad ${ }^{2}$, Muhammad Khan Asif $^{1}$, Zamri Radzi ${ }^{3}$, \\ Zuraiza Mohamad Zaini ${ }^{1}$ and Hairil Rashmizal Abdul Razak ${ }^{4}$
}

\begin{abstract}
Background: Cone Beam Computed Tomography $(C B C T)$ is a reliable radiographic modality to assess trabecular bone microarchitecture. The aim of this study was to determine the effect of CBCT image reconstruction parameters, namely, the threshold value and reconstruction voxel size, on trabecular bone microstructure assessment.

Methods: Five sectioned maxilla of adult Dorper male sheep were scanned using a CBCT system with a resolution of $76 \mu^{3}$ (Kodak 9000). The CBCT images were reconstructed using different reconstruction parameters and analysed. The effect of reconstruction voxel size $\left(76,100\right.$ and $200 \mu^{3}$ ) and threshold values $( \pm 15 \%$ from the global threshold value) on trabecular bone microstructure measurement was assessed using image analysis software (CT analyser version 1.15).

Results: There was no significant difference in trabecular bone microstructure measurement between the reconstruction voxel sizes, but a significant difference $(\mathrm{Tb} . \mathrm{N}=0.03, \mathrm{~Tb} . \mathrm{Sp}=0.04, \mathrm{~Tb} . \mathrm{Th}=0.01, \mathrm{BV} / \mathrm{TV}=0.00$ ) was apparent when the global threshold value was decreased by $15 \%$.

Conclusions: Trabecular bone microstructure measurements are not compromised by changing the CBCT reconstruction voxel size. However, measurements can be affected when applying a threshold value of less than $15 \%$ of the recommended global value.
\end{abstract}

Keywords: CBCT, Trabecular bone, Reconstruction parameters, Threshold value

\section{Background}

Assessment of trabecular bone microstructure from $\mathrm{CBCT}$ images prior to implant surgery is a growing treatment planning protocol to ensure successful placement and clinical longevity of the dental implants [1]. This is because the trabecular bone microstructure has been shown to have major effects on primary stability during implant placement $[2,3]$. In addition, primary stability can be accurately predicted based on the status of the trabecular bone microarchitecture prior to implant placement [4]. Nevertheless, the assessment of trabecular parameters from CBCT images may be compromised by the reconstruction parameters that are used for

\footnotetext{
* Correspondence: norlizaibrahim@um.edu.my

${ }^{1}$ Department of Oral and Maxillofacial Clinical Sciences, Faculty of Dentistry,

University of Malaya, 50603 Kuala Lumpur, Malaysia

Full list of author information is available at the end of the article
}

image segmentation, such as the reconstruction voxel size and threshold value.

Reconstruction voxel size is the voxel size that is used to reconstruct an image of raw data. A larger image reconstruction voxel size than the actual image acquisition voxel size is usually used to reduce the reconstruction time and to reduce computational expenses [5]. This is used in some practices such as analysing large-scale finite element models using a micro- CT-based image [5-7]. However, it is presumed that the image accuracy will be decreased when using a larger reconstruction voxel size than the acquisition voxel size [8]. The effect of CBCT reconstruction voxel size has focused on image quality [9] and 3D measurement of dental morphology [10]. However, the effect on trabecular bone microstructure measurements remains unknown.

(c) The Author(s). 2020 Open Access This article is distributed under the terms of the Creative Commons Attribution 4.0 International License (http://creativecommons.org/licenses/by/4.0/), which permits unrestricted use, distribution, and reproduction in any medium, provided you give appropriate credit to the original author(s) and the source, provide a link to the Creative Commons license, and indicate if changes were made. The Creative Commons Public Domain Dedication waiver (http://creativecommons.org/publicdomain/zero/1.0/) applies to the data made available in this article, unless otherwise stated. 


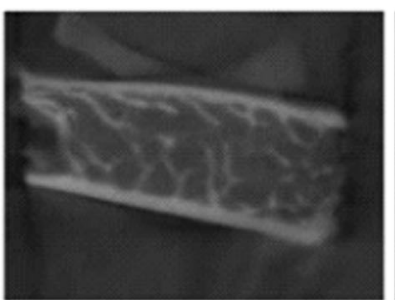

$\mathrm{V} 1=76 \mu \mathrm{m}^{3}$

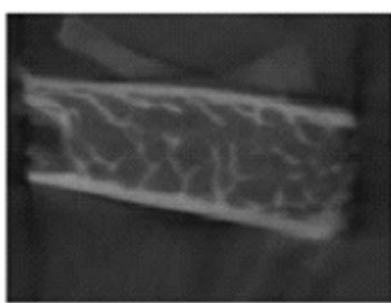

$\mathrm{V} 2=100 \mu \mathrm{m}^{3}$

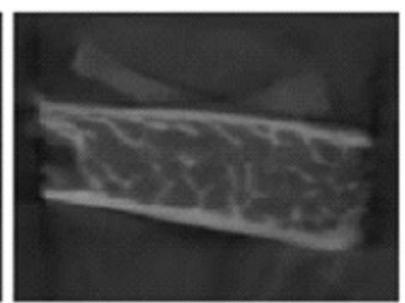

$\mathrm{V} 3=200 \mu \mathrm{m}^{3}$

Fig. 1 Three reconstructed images using voxel size of $76 \mu \mathrm{m}^{3}$ (V1), $100 \mu \mathrm{m}^{3}$ (V2) and $200 \mu \mathrm{m}^{3}$ (V3) were binarised and analysed using CT-Analyser software

Segmentation is a process of separating images to either bone or other soft tissue structures which can be affected by the threshold values [11]. Many studies have used $\mu \mathrm{CT}$ to investigate the effect of threshold values on trabecular microstructure parameters such as trabecular number (Tb.N), thickness (Tb.Th), spacing (Tb.Sp) and bone volume fraction (BV/TV) of small animal models [12]. Although studies on scanning parameters are abundant in large animal models, the effect of reconstruction parameters using CBCT are scarce. Hence, as it has been suggested that СВCT can be used for trabecular bone measurement to aid surgical treatment planning, the CBCT reconstruction parameters that affect these measurements should be investigated.

Threshold values are commonly used for segmentation as it is the most straightforward and feasible segmentation method specifically when measuring bone volume [13]. Therefore, its effect on trabecular quantification is also investigated in this study. The findings of this study will provide clinicians with an evidence-based guideline in deciding the suitable reconstruction voxel size and threshold values to be used for their CBCT image reconstruction prior to trabecular bone microstructure analysis.

\section{Methods}

Five sectioned maxillae of adult Dorper male sheep (age ranging from 20 to 48 months and body weight
50-60 kg) were obtained from the Animal Experimental Unit, Faculty of Veterinary Medicine, University Putra Malaysia (UPM), Malaysia following approval from the Institutional Animal Care and Use Committee (IACUC), UPM (No. UPM/IACUC/AUP-R031). The samples were imaged using a CBCT system (Carestream 9000, Kodak) at the Division of Oral Radiology, Faculty of Dentistry, University of Malaya, Kuala Lumpur, Malaysia. The selected scanning parameters for CBCT were $76 \mu \mathrm{m}^{3}$ for the voxel size, $4 \times 4 \mathrm{~cm}$ FOV and $360^{\circ}$ arm rotation. Images were acquired at $65 \mathrm{kv}, 6 \mathrm{~mA}$ and $10.8 \mathrm{~s}$. The samples were fitted in a cylinder-shaped plastic container to reduce any possible movement during scanning. The images were subsequently converted into BMP and imported into DataViewer (v 1.5, SkyScan, Kontich, Belgium) to obtain the sagittal view for image processing and analyses in CTAnalyser software (v 1.15, SkyScan, Kontich, Belgium).

The effect of CBCT reconstruction voxel size on trabecular bone microstructure assessment

The images were reconstructed using three different voxel sizes $\left(76,100\right.$, and $\left.200 \mu \mathrm{m}^{3}\right)$ resulting in three different datasets. For the first dataset, a ROI was selected $(14 \times 8 \mathrm{~mm})$ and reconstructed using a voxel size of $76 \mu \mathrm{m}^{3}$. Then, the same ROI was used to reconstruct the other 2 datasets using a voxel size of 100 and $200 \mu \mathrm{m}^{3}$, respectively (Fig. 1). Thus, three sets of ROI were

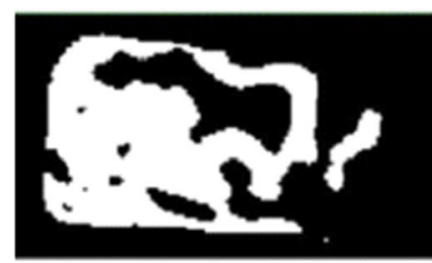

$\mathrm{TH} 1=69$

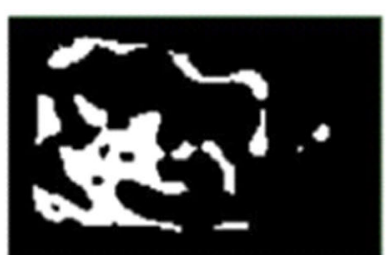

$\mathrm{TH} 2=82$

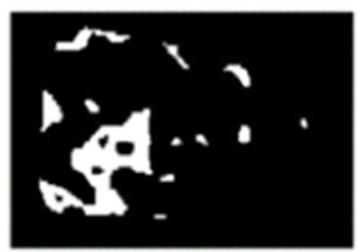

TH3 $=94$

Fig. 2 Images were thresholded +15\% from the global threshold value (69, 82: global threshold value and 94) and binarised in the CTAnalyser software 
obtained which were thresholded and binarized using a global threshold value (threshold value 82). The global threshold value was automatically generated by CTAnalyser software (v 1.15, SkyScan, Kontich, Belgium). This global threshold value was used as the optimal threshold value in this study. Later, trabecular bone analysis was performed using CTAnalyser software (v 1.15, SkyScan, Kontich, Belgium) to examine the effect of various reconstruction voxel sizes on the assessment of trabecular bone parameters (Tb.N, Tb.Th, Tb.Sp and BV/TV).

\section{The effect of $C B C T$ threshold value on trabecular bone microstructure assessment}

The effect of different range of threshold values on the CBCT datasets was assessed using the first dataset as a reference. Three datasets were created by varying the global threshold value as follows: (Dataset A) decreasing the global selected threshold value by $15 \%$, (Dataset B) and (Dataset $\mathrm{C}$ ) increasing the global threshold value by 15\% (Fig. 2). The images from all 3 datasets were then exported into CTAnalyser software (v 1.15, SkyScan, Kontich, Belgium) for the selection of the ROI. Threedimensional analyses were then performed on all three datasets to assess the trabecular bone parameters (Tb.N, Tb.Th, Tb.Sp and BV/TV).

\section{Statistical analyses}

The assessments were performed twice by two examiners with a minimum interval of one week between the two measurements. Intraclass correlation coefficient (ICC) was employed to examine the intra-observer reliability and inter-rater agreement in reproducing the measurements. The trabecular bone microstructure parameters that were assessed include trabecular number (Tb.N), thickness (Tb.Th), separation (Tb.Sp) and bone volume fraction (BV/TV). One-way ANOVA was used to assess the differences obtained from three reconstruction voxel sizes and threshold values. Additionally, the Bonferroni test was used as a post hoc test to examine the significant differences between threshold values.

\section{Results}

The effect of reconstruction voxel size on the assessment of trabecular bone microstructure

The intra-observer reproducibility $(>0.91)$ and inter-rater agreement $(>0.80)$ of trabecular bone microstructure measurements were excellent for all parameters (Tables 1 and 2). One-way ANOVA showed no significant difference between the three reconstruction voxel sizes in all trabecular bone microstructures (Tb.N, $p=0.579$; Tb.Th, $p=0.095$; Tb.Sp, $p=0.131$; BV/TV, $p=0.908)$, as shown in Table 3.
Table 1 Intraobserver reliability for different reconstruction voxel sizes using the intraclass correlation coefficient (ICC)

\begin{tabular}{llll}
\hline & $\mathrm{V} 1=76 \mu \mathrm{m}^{3}$ & $\mathrm{~V} 2=100 \mu \mathrm{m}^{3}$ & $\mathrm{~V} 3=200 \mu \mathrm{m}^{3}$ \\
\hline Tb.N & 0.991 & 0.986 & 0.977 \\
Tb.Sp & 0.912 & 0.973 & 0.989 \\
Tb.Th & 0.981 & 0.989 & 0.996 \\
BV/TV & 0.994 & 0.999 & 0.999 \\
\hline
\end{tabular}

Trabecular number (Tb.N), separation (Tb.Sp), thickness (Tb.Th), and bone volume fraction (BV/TV)

$\mathrm{V}=$ Voxel size

${ }^{*} p<0.05$

\section{The effect of different threshold values on trabecular} bone microstructure measurements

The reproducibility of trabecular bone microstructure measurements revealed excellent intra-observer reliability $(>0.91)$ and inter-rater agreement $(>0.82)$ when using different threshold values (Tables 4 and 5). Oneway ANOVA showed significant differences between the three tested threshold values. Bonferroni Post-Hoc analyses with pair-wise multiple comparisons were performed to test the difference between the three different threshold values. A significant difference was observed $(\mathrm{Tb} . N=0.03, \mathrm{~Tb} . \mathrm{Sp}=0.04, \mathrm{~Tb} . \mathrm{Th}=0.01, \mathrm{BV} / \mathrm{TV}=0.00)$ when the threshold value was decreased by $15 \%$ from the global value. However, the increase in the threshold value (Th: 90) from the global value (Th: 82$)$ had no significant $(p>0.05)$ effect on the trabecular bone measurements (Table 6).

\section{Discussion}

The enhanced resolution of cone-beam computed tomography $(\mathrm{CBCT})$ images has significantly improved the measurement of trabecular bone microstructure [14, 15]. Unlike scanning parameters (voxel size, field of view and scanning rotation) [16], the effect of CBCT reconstruction parameters (reconstruction voxel size and threshold

Table 2 Inter-rater agreement for different reconstruction voxel sizes using the intraclass correlation coefficient (ICC)

\begin{tabular}{llll}
\hline & $\mathrm{V} 1=76 \mu \mathrm{m}^{3}$ & $\mathrm{~V} 2=100 \mu \mathrm{m}^{3}$ & $\mathrm{~V} 3=200 \mu \mathrm{m}^{3}$ \\
\hline Tb.N & 0.823 & 0.840 & 0.813 \\
Tb.Sp & 0.804 & 0.853 & 0.876 \\
Tb.Th & 0.883 & 0.819 & 0.837 \\
BV/TV & 0.829 & 0.871 & 0.845 \\
\hline
\end{tabular}

Trabecular number (Tb.N), separation (Tb.Sp), thickness (Tb.Th), and bone volume fraction (BV/TV)

$V=$ Voxel size

${ }^{*} p<0.05$ 
Table 3 The mean and standard deviation (SD) of trabecular microstructure measurements analyzed using One Way ANOVA for different reconstruction voxel size

\begin{tabular}{|c|c|c|c|c|c|c|c|c|c|c|c|c|c|}
\hline \multirow{2}{*}{$\begin{array}{l}\text { Voxel } \\
\text { size }\end{array}$} & \multirow[t]{2}{*}{$\mathrm{n}$} & \multicolumn{3}{|c|}{ Trabecular number Tb.N $\left(\mu \mathrm{m}^{-1}\right)$} & \multicolumn{3}{|c|}{ Trabecular spacing Tb.Sp $(\mu \mathrm{m})$} & \multicolumn{3}{|c|}{ Trabecular thickness Tb.Th ( $\mu m)$} & \multicolumn{3}{|c|}{ Bone Volume/Tissue volume BV/TV \% } \\
\hline & & Mean & SD & $p$ & Mean & SD & $p$ & Mean & SD & $p$ & Mean & SD & $p$ \\
\hline $76 \mu m^{3}$ & 5 & 0.0153 & 0.007 & 0.579 & 17.6388 & 2.040 & 0.095 & 6.8204 & 1.832 & 0.131 & 11.4783 & 8.212 & 0.908 \\
\hline $100 \mu \mathrm{m}^{3}$ & 5 & 0.0168 & 0.008 & & 16.8204 & 2.206 & & 6.1350 & 1.966 & & 11.6022 & 8.817 & \\
\hline $200 \mu m^{3}$ & 5 & 0.0204 & 0.007 & & 14.4790 & 2.230 & & 4.3543 & 1.676 & & 9.6478 & 6.309 & \\
\hline
\end{tabular}

${ }^{*} p<0.05$

value) on diagnostic accuracy of trabecular bone microstructure has not been reported $[17,18]$.

The accuracy of micro-CT images in measuring trabecular bone microstructure can be compromised by the reconstruction voxel size $[8,19,20]$. Previous micro-CT studies have reported that the image quality cannot be improved by using a smaller reconstruction voxel size after originally scanning the sample using a large voxel size $[8,21]$. This is due to an increase in image noise (9). Therefore, in the current study, CBCT images that were scanned using $76 \mu \mathrm{m}^{3}$ were reconstructed using larger voxel sizes $\left(100\right.$ and $\left.200 \mu \mathrm{m}^{3}\right)$. The findings indicated no differences in trabecular bone measurements between different reconstructed images. However, our results differ to those reported in previously studies $[8,21]$ as the scanning voxel size $\left(76 \mu \mathrm{m}^{3}\right)$ is almost 4 times larger than was used in micro-CT studies $\left(21 \mu \mathrm{m}^{3}\right)$. It was demonstrated in micro-CT studies that only certain trabecular bone parameters namely $\mathrm{BV} / \mathrm{TV}$ and $\mathrm{Tb}$.Th are compromised by scanning parameters when reconstructing the images of $21 \mu^{3}$ using larger voxel sizes (50 and $110 \mathrm{~m}^{3}$ ) [8]. This is due to the fact that the trabecular bone parameters are significantly affected by the scanning voxel size rather than the reconstruction voxel size. However, this effect can only be observed when the difference between the scan and reconstruction voxel is very large [21]. The results of the present study showed that trabecular bone microstructure measurements are not influenced by CBCT reconstruction voxel size, although it is important to note other $\mathrm{CBCT}$

Table 4 Intraobserver reliability for different threshold values using intraclass correlation coefficient (ICC)

\begin{tabular}{llll}
\hline & Th $1=73$ & Th $2=82$ & Th $3=90$ \\
\hline Tb.N & 0.988 & 0.991 & 0.994 \\
Tb.Sp & 0.996 & 0.912 & 0.975 \\
Tb.Th & 0.998 & 0.981 & 0.973 \\
BV/TV & 0.971 & 0.994 & 0.985
\end{tabular}

Trabecular number (Tb.N), separation (Tb.Sp), thickness (Tb.Th), bone volume fraction (BV/TV) and threshold value (Th) systems with different scanning and reconstruction parameters may generate different results.

Threshold values may influence the analysis of trabecular microstructure parameters in micro-CT images [22-24]. Similarly, our study of CBCT images showed that a reducing the threshold value by $15 \%$ had a significant effect on all trabecular bone microstructure parameters $(\mathrm{Tb} . N=0.03, \mathrm{~Tb} . \mathrm{Sp}=0.04, \mathrm{~Tb} . \mathrm{Th}=0.01, \mathrm{BV} / \mathrm{TV}=$ $0.00)$. However, increasing the threshold value from the global value had no significant $(p<0.05)$ effect on trabecular bone measurements (Table 4). The deviation of trabecular bone measurements might be due to partial volume effects that might alter the layers of voxels from the trabecular surface [25]. However, Tb.N measurements are insensitive to threshold variation if the selected threshold values are within the range of realistic values.

However, some limitations should be noted. In this study, the samples were not assessed using micro CT images due to computational constrains. Although strong correlations between micro $\mathrm{CT}$ and $\mathrm{CBCT}$ trabecular bone measurements have been largely described [26-28], the comparison in varying the reconstruction parameters is highly recommended to further validate the accuracy of the current findings.

\section{Conclusion}

This study showed that trabecular bone microstructure measurements are not compromised by changing the $\mathrm{CBCT}$ reconstruction voxel size. However,

Table 5 Inter-rater agreement for different threshold values using intraclass correlation coefficient (ICC)

\begin{tabular}{llll}
\hline & Th $1=73$ & Th $2=82$ & Th $3=90$ \\
\hline Tb.N & 0.870 & 0.853 & 0.824 \\
Tb.Sp & 0.857 & 0.841 & 0.872 \\
Tb.Th & 0.865 & 0.821 & 0.854 \\
BV/TV & 0.837 & 0.860 & 0.883 \\
\hline
\end{tabular}

Trabecular number (Tb.N), separation (Tb.Sp), thickness (Tb.Th), bone volume fraction (BV/TV) and threshold value (Th) 
Table 6 The mean and standard deviation (SD) of trabecular microstructural measurements analyzed using One Way ANOVA for different threshold values

\begin{tabular}{|c|c|c|c|c|c|c|c|c|c|c|c|c|c|}
\hline \multirow[t]{2}{*}{$\begin{array}{l}\text { Threshold } \\
\text { value }\end{array}$} & \multirow[t]{2}{*}{$n$} & \multicolumn{3}{|c|}{$\begin{array}{l}\text { Trabecular } \\
\text { number Tb.N }\left(\mu m^{-1}\right)\end{array}$} & \multicolumn{3}{|c|}{$\begin{array}{l}\text { Trabecular spacing } \\
\text { Tb.Sp ( } \mu \mathrm{m})\end{array}$} & \multicolumn{3}{|c|}{$\begin{array}{l}\text { Trabecular thickness } \\
\text { Tb.Th }(\mu \mathrm{m})\end{array}$} & \multicolumn{3}{|c|}{$\begin{array}{l}\text { Bone Volume/Tissue } \\
\text { volume BV/TV \% }\end{array}$} \\
\hline & & Mean & SD & $P$ & Mean & SD & $p$ & Mean & SD & $p$ & Mean & SD & $p$ \\
\hline 73 & 5 & 0.0264 & 0.00385 & & 14.4607 & 1.49057 & & 12.2463 & 3.12846 & & 32.7632 & 10.87921 & \\
\hline 82 & & & & 0.033 & & & 0.037 & & & 0.007 & & & 0.004 \\
\hline 90 & & & & 0.000 & & & 0.001 & & & 0.000 & & & 0.000 \\
\hline 82 & 5 & 0.0153 & 0.00784 & & 17.6388 & 2.04041 & & 6.8204 & 1.83221 & & 11.4783 & 8.21205 & \\
\hline 73 & & & & 0.033 & & & 0.037 & & & 0.007 & & & 0.004 \\
\hline 90 & & & & 0.082 & & & 0.190 & & & 0.381 & & & 0.404 \\
\hline 90 & 5 & 0.0059 & 0.00518 & & 19.8544 & 1.55221 & & 4.4882 & 1.42399 & & 3.2503 & 3.42096 & \\
\hline 73 & & & & 0.000 & & & 0.001 & & & 0.000 & & & 0.000 \\
\hline 82 & & & & 0.82 & & & 0.190 & & & 0.381 & & & 0.404 \\
\hline
\end{tabular}

${ }^{*} p<0.05$

measurements can be affected when applying a threshold value less than $15 \%$ of the recommended global value.

\section{Abbreviations}

BV/TV: Bone volume fraction; CBCT: Cone beam computed tomography; Micro-CT: Micro- computed tomography; Tb.N: Trabecular number;

Tb.Sp: Trabecular spacing; Tb.Th: Trabecular thickness

\section{Acknowledgements}

Not applicable.

\section{Authors' contributions}

AMAM performed the Data analysis and interpretation. NI designed and supervised the study. NI also prepared the manuscript draft. RA designed and supervised the study. RA also prepared the manuscript draft. MK assisted in drafting the manuscript, finalising the manuscript and data analysis. ZR conducted critical analysis and drafting of the manuscript. ZMZ drafted the manuscript, analysed the data. HRAR drafted the manuscript and final approval. All authors read and approved the final manuscript.

\section{Funding}

This study was funded by Universiti Teknologi Mara (UiTM), Malaysia Higher Education Fundamental Research Grant Scheme (FRGS/1/2015/SKK14/UITM 02/2) and High Impact Research MoE Grant (UM.C/625/1/HIR/MOHE/DENT/

21) by Ministry of Higher Education, Malaysia.

\section{Availability of data and materials}

The datasets used and/or analysed during the current study are available from the corresponding author on reasonable request.

\section{Ethics approval and consent to participate}

Ethical approval for the study was obtained from the Institutional Animal Care and Use Committee (IACUC), UPM (No. UPM/IACUC/AUP-R031).

\section{Consent for publication}

Not applicable.

\section{Competing interests}

The authors declare that they have no competing interests.

\section{Author details}

'Department of Oral and Maxillofacial Clinical Sciences, Faculty of Dentistry, University of Malaya, 50603 Kuala Lumpur, Malaysia. ${ }^{2}$ Faculty of Dentistry, Universiti Teknologi MARA, Sungai Buloh Campus, Jalan Hospital 47000 Sungai Buloh, Shah Alam, Selangor, Malaysia. ${ }^{3}$ Department of Paediatric Dentistry and Orthodontics, Faculty of Dentistry, University of Malaya, 50603
Kuala Lumpur, Malaysia. ${ }^{4}$ Centre for Diagnostic Nuclear Imaging, Universiti Putra Malaysia, 43400 Serdang, Selangor, Malaysia.

Received: 1 November 2019 Accepted: 4 February 2020 Published online: 10 February 2020

\section{References}

1. DeTolla DH, Andreana S, Patra A, Buhite R, Comella B. The role of the finite element model in dental implants. J Oral Implantol. 2000:26:77-81.

2. Sakka S, Coulthard P. Bone quality: a reality for the process of osseointegration. Implant Dent. 2009:18:480-5.

3. Fanuscu Ml, Chang TL. Three-dimensional morphometric analysis of human cadaver bone: microstructural data from maxilla and mandible. Clin Ora Implants Res. 2004;15:213-8.

4. Ibrahim N, Parsa A, Hassan B, Van der Stelt P, Wismeijer D. Diagnostic imaging of trabecular bone microstructure for oral implants: a literature review. Dentomaxillofac Radiol. 2013;42:20120075.

5. Homminga J, Huiskes $R$, Van Rietbergen $B$, Rüegsegger $P$, Weinans $H$. Introduction and evaluation of a gray-value voxel conversion technique. J Biomech. 2001;34(4):513-7.

6. Ladd AJ, Kinney JH, Haupt DL, Goldstein SA. Finite-element modeling of trabecular bone: comparison with mechanical testing and determination of tissue modulus. J Orthop Res. 1998;16:622-8.

7. Van Rietbergen $B$, Müller R, Ulrich D, Rüegsegger P, Huiskes R. Tissue stresses and strain in trabeculae of a canine proximal femur can be quantified from computer reconstructions. J Biomech. 1999:32:165-73.

8. Kim D-G, Christopherson GT, Dong XN, Fyhrie DP, Yeni YN. The effect of microcomputed tomography scanning and reconstruction voxel size on the accuracy of stereological measurements in human cancellous bone. Bone. 2004;35:1375-82.

9. Tanimoto $\mathrm{H}$, Arai $\mathrm{Y}$. The effect of voxel size on image reconstruction in cone-beam computed tomography. Oral Radiol. 2009;25:149.

10. Hassan B, Souza PC, Jacobs R, de Azambuja BS, van der Stelt P. Influence of scanning and reconstruction parameters on quality of three-dimensional surface models of the dental arches from cone beam computed tomography. Clin Oral Investig. 2010;14:303-10.

11. Sharma N, Aggarwal LM. Automated medical image segmentation techniques. J Med Phys. 2010;35:3.

12. Christiansen BA. Effect of micro-computed tomography voxel size and segmentation method on trabecular bone microstructure measures in mice. Bone reports. 2016:5:136-40.

13. Ulrich $D$, van Rietbergen $B$, Weinans $H$, Rüegsegger $P$. Finite element analysis of trabecular bone structure: a comparison of image-based meshing techniques. J Biomech. 1998:31:1187-92.

14. Miracle A, Mukherji S. Conebeam CT of the head and neck, part 2: clinical applications. AJNR Am J Neuroradiol. 2009:30:1285-92.

15. Koskinen SK, Haapamäki W, Salo J, Lindfors NC, Kortesniemi M, Seppälä L, et al. CT arthrography of the wrist using a novel, mobile, dedicated extremity cone-beam CT (CBCT). Skelet Radiol. 2013;42:649-57. 
16. Bechara B, McMahan CA, Moore WS, Noujeim M, Geha H, Teixeira FB. Contrast-to-noise ratio difference in small field of view cone beam computed tomography machines. J Oral Sci. 2012;54:227-32.

17. Pauwels R, Faruangsaeng T, Charoenkarn T, Ngonphloy N, Panmekiate S. Effect of exposure parameters and voxel size on bone structure analysis in CBCT. Dentomaxillofac Radiol. 2015;44:20150078.

18. Maret D, Telmon N, Peters O, Lepage B, Treil J, Inglèse J, et al. Effect of voxel size on the accuracy of $3 \mathrm{D}$ reconstructions with cone beam $\mathrm{CT}$. Dentomaxillofac Radiol. 2012:41:649-55.

19. Cooper D, Turinsky A, Sensen C, Hallgrimsson B. Effect of voxel size on 3D micro-CT analysis of cortical bone porosity. Calcif Tissue Int. 2007;80:211-9.

20. Tabor Z. Analysis of the influence of image resolution on the discriminating power of trabecular bone architectural parameters. Bone. 2004;34:170-9.

21. Yeni YN, Christopherson GT, Dong XN, Kim D-G, Fyhrie DP. Effect of microcomputed tomography voxel size on the finite element model accuracy for human cancellous bone. J Biomech Eng. 2005;127:1-8.

22. Waarsing JH, Day JS, Weinans H. An improved segmentation method for in vivo $\mu C T$ imaging. J Bone Miner Res. 2004;19:1640-50.

23. Ito M, Nakamura T, Matsumoto T, Tsurusaki K, Hayashi K. Analysis of trabecular microarchitecture of human iliac bone using microcomputed tomography in patients with hip arthrosis with or without vertebral fracture. Bone. 1998:23:163-9.

24. Rüegsegger P, Koller B, Müller R. A microtomographic system for the nondestructive evaluation of bone architecture. Calcif Tissue Int. 1996;58:24-9.

25. Hara T, Tanck E, Homminga J, Huiskes R. The influence of microcomputed tomography threshold variations on the assessment of structural and mechanical trabecular bone properties. Bone. 2002;31:107-9.

26. Ibrahim N, Parsa A, Hassan B, van der Stelt P, Aartman IH, Wismeijer D. Accuracy of trabecular bone microstructural measurement at planned dental implant sites using cone-beam CT datasets. Clin Oral Implants Res. 2014;25:941-5

27. Gonzalez-Garcia R, Monje F. The reliability of cone-beam computed tomography to assess bone density at dental implant recipient sites: a histomorphometric analysis by micro-CT. Clin Oral Implants Res. 2013;24:871-9.

28. Van Dessel J, Huang Y, Depypere M, Rubira-Bullen I, Maes F, Jacobs R. A comparative evaluation of cone beam CT and micro-CT on trabecular bone structures in the human mandible. Dentomaxillofac Radiol. 2013:42: 20130145.

\section{Publisher's Note}

Springer Nature remains neutral with regard to jurisdictional claims in published maps and institutional affiliations.

Ready to submit your research? Choose BMC and benefit from:

- fast, convenient online submission

- thorough peer review by experienced researchers in your field

- rapid publication on acceptance

- support for research data, including large and complex data types

- gold Open Access which fosters wider collaboration and increased citations

- maximum visibility for your research: over $100 \mathrm{M}$ website views per year

At $\mathrm{BMC}$, research is always in progress.

Learn more biomedcentral.com/submissions 